\title{
THE ABUNDANCE SCATTER IN M33 FROM H II REGIONS: IS THERE ANY EVIDENCE FOR AZIMUTHAL METALLICITY VARIATIONS? ${ }^{1}$
}

FABIO BRESOLIN

Institute for Astronomy, 2680 Woodlawn Drive, Honolulu, HI 96822, USA

\begin{abstract}
Optical spectra of $25 \mathrm{H}$ II regions in the inner two kpc of the M33 disk have been obtained with the GMOS spectrograph at the Gemini North telescope. The oxygen abundance gradient measured from the detection of the [O III] $\lambda 4363$ auroral line displays a scatter of approximately 0.06 dex, a much smaller value than recently reported by Rosolowsky \& Simon in this galaxy. The analysis of the abundances for a large sample of H II regions derived from the $\mathrm{R}_{23}$ strong-line indicator confirms that the scatter is small over the full disk of M33, consistent with the measuring uncertainties, and comparable to what is observed in other spiral galaxies. No evidence is therefore found for significant azimuthal variations in the present-day metallicity of the interstellar medium in this galaxy on spatial scales from $\sim 100 \mathrm{pc}$ to a few kpc. A considerable fraction of M33 H II regions with auroral line detections show spectral features revealing sources of hard ionizing radiation (such as $\mathrm{He}$ II emission and large [Ne III], [O III] line fluxes). Since $\mathrm{R}_{23}$ is shown to severely underestimate the oxygen abundances in such cases, care must be taken in chemical abundance studies of extragalactic $\mathrm{H}$ II regions based on this strong-line indicator.
\end{abstract}

Subject headings: galaxies: abundances — galaxies: ISM — galaxies: individual (M33)

\section{INTRODUCTION}

The occurrence of radial metallicity gradients in spiral galaxies is presently understood within the inside-out formation paradigm as due to disk growth by means of radiallydependent gas infall and star formation rate (Matteucci \& Francois 1989, Boissier \& Prantzos 1999). Recent age estimates of resolved stellar populations as a function of galactocentric distance in nearby galaxies has provided convincing evidence for the inside-out evolution of disk galaxies (Williams et al. 2009; Gogarten et al. 2010). At the same time, the study of present-day metallicity gradients in galaxies from H II region spectroscopy (Vila-Costas \& Edmunds 1992: Zaritsky et al. 1994, Kennicutt et al. 2003) continues to provide important empirical constraints for the complex set of parameters that describe galactic chemical evolution models (Yin et al. 2009, Fu et al. 2009).

Azimuthal variations in the chemical composition of external spiral galaxies are observationally much less constrained than radial gradients. The mixing of the interstellar medium (ISM) occurs over a range of timescales, that grow with the extent of the spatial scale considered. On scales between 0.1 and $1 \mathrm{kpc}$ the effects of cloud collisions and expanding supershells, powered by stellar winds and supernova explosions, and combined with galactic differential rotation, lead to heavy element dispersal and mixing of the ISM on relatively short timescales, around $10^{8}$ yr (Roy \& Kunth 1995), which helps to explain the high degree of chemical homogeneity observed in the ISM of galaxies (Scalo \& Elmegreen 2004; Edmunds 2005). In the case of the spiral galaxy M101, Kennicutt \&

${ }^{1}$ Based on observations obtained at the Gemini Observatory, which is operated by the Association of Universities for Research in Astronomy, Inc., under a cooperative agreement with the NSF on behalf of the Gemini partnership: the National Science Foundation (United States), the Science and Technology Facilities Council (United Kingdom), the National Research Council (Canada), CONICYT (Chile), the Australian Research Council (Australia), Ministério da Ciência e Tecnologia (Brazil) and Ministerio de Ciencia, Tecnología e Innovación Productiva (Argentina).
Garnett (1996) concluded that the local scatter in metallicity is significantly smaller than the $0.1-0.15$ dex dispersion derived from the oxygen abundances of $41 \mathrm{H}$ II regions, a conclusion that is in agreement with the expected level of inhomogeneity of the ISM from hierarchical models of star formation $(\sim 0.05$ dex, Elmegreen (1998).

More recently, Bresolin et al. (2009) measured the oxygen abundances of $28 \mathrm{HII}$ regions in NGC 300 using the $[\mathrm{O}$ III $] \lambda 4363$ auroral line as a temperature diagnostic, and found a scatter in the radial gradient of only 0.05 dex. On the other hand, in M33 Rosolowsky \& Simon (2008) found a substantial intrinsic fluctuation in the H II region oxygen abundance, $\sim 0.11$ dex, in addition to the scatter that is simply due to observational errors. In particular, in the central two kpc of the M33 disk the dispersion in $\mathrm{O} / \mathrm{H}$ is $0.2 \mathrm{dex}$, with a peak-topeak variation of 0.8 dex over a spatial scale of about $1 \mathrm{kpc}$. This result appears difficult to reconcile with the short mixing timescale of the ISM and with the small scatter measured in the present-day metallicities of other nearby spirals.

In this paper a new sample of $\mathrm{H}$ II regions located in the inner two kpc of M33 is presented, with the goal of verifying the substantial local dispersion in the oxygen abundance detected by Rosolowsky \& Simon (2008, = RS08). Measuring accurate $\mathrm{H}$ II region abundances in the central regions of spiral galaxies is critical for radial gradient studies, but it is generally made difficult by the enhanced cooling resulting from the increased metal line emission, which hinders the detection of the weak electron temperature diagnostic lines, such as [O III] $\lambda 4363$ (Bresolin 2008). The observations presented here alleviate this difficulty, thanks to high-quality detections of the $[\mathrm{O}$ III] $\lambda 4363$ line in $\mathrm{H}$ II regions with galactocentric distances as small as $0.2 \mathrm{kpc}$ (Sect. 2). The chemical abundance analysis is carried out complementing the new data with existing samples of $\mathrm{H}$ II regions, using both the auroral line information and the $\mathrm{R}_{23}$ strong-line abundance indicator (Sect 3). The systematic effect on $\mathrm{R}_{23}$-based abundances induced by the hard ionizing radiation field detected in several 
of the M33 H II regions is discussed in Sec. 4.

\section{OBSERVATIONS}

Spectra of H II regions in M33 were obtained with the Gemini Multi-Object Spectrograph (GMOS, Hook et al. 2004) at the Gemini North facility. The targets were selected from narrow-band $\mathrm{H} \alpha$ images of two 5.5 $\times 5.5$ GMOS fields in the inner parts of the galaxy, centered approximately 2!1 (0.83 kpc deprojected distance) $\mathrm{W}$ and 5.4 (1.67 kpc) $\mathrm{S}$ of the center. The spectroscopic data were acquired in queue mode on October 17, 2009, using two multi-object masks, one per field, with 1. . 2 -wide slits. The seeing conditions were $\sim 0$ ! $^{\prime \prime}$ during the observations of the $\mathrm{W}$ field, and $\sim 1$ !' 2 for the $\mathrm{S}$ field. In order to minimize the effects of the differential atmospheric refraction the data were acquired at airmasses smaller than 1.16. Three $1800 \mathrm{~s}$ exposures were secured for each of the two fields using the B600 grating, which provided spectra covering the $3500-5100 \AA$ wavelength range at a spectral resolution of $\sim 5.5 \AA$. For some of the targets, depending on their spatial distribution, the spectral coverage extended up to $\sim 6000 \AA$.

IRAF $^{2}$ routines in the gemini/gmos package were used for electronic bias subtraction, flat field correction and wavelength calibration of the raw data frames. Observations of the spectrophotometric standard star Wolf 1346 yielded the flux calibration. The final version of the spectra was obtained by averaging the three spectra corresponding to each individual slit. The H II region sample comprises 25 objects, whose locations are shown in Fig. 1. The celestial coordinates of the targets are summarized in Table 1 (where objects are listed in order of decreasing declination), together with their galactocentric distances in $\mathrm{kpc}$, deprojected adopting an inclination angle for M33 of 56 degrees (Zaritsky et al. 1989), a position angle of the major axis of 23 degrees (de Vaucouleurs et al. 1991), and the center coordinates measured by Massey et al. (1996). A distance of $840 \mathrm{kpc}$ from Freedman et al. (2001) was assumed. The identification of the subcomponents of the $\mathrm{H}$ II regions given in column (5) of Table 1 was taken from Hodge et al. (2002).

\subsection{Line intensities and oxygen abundances}

The emission line intensities were measured with the splot program in IRAF by integrating the fluxes under the line profiles. The line intensities were corrected for interstellar reddening by assuming a case $\mathrm{B}$ intrinsic $\mathrm{H} \gamma / \mathrm{H} \beta$ ratio of 0.47 at $T_{e}=10^{4} \mathrm{~K}$ and the Seaton (1979) reddening law. The effects of the underlying stellar populations on the strength of the Balmer lines were accounted for by requiring that the $\mathrm{H} \gamma / \mathrm{H} \beta$ and $\mathrm{H} \delta / \mathrm{H} \beta$ line ratios provided the same value for the extinction. A median equivalent width of $2 \AA$ was found for the absorption component, and was applied to the whole sample. The resulting reddening-corrected emission line fluxes, normalized to $\mathrm{H} \beta=100$, are presented in Table 2 . The errors account for uncertainties in the flat fielding, the flux calibration, the level of the continuum in proximity of each line and the logarithmic extinction coefficient $c(\mathrm{H} \beta)$.

The electron temperature $\left(T_{e}\right)$ diagnostic line [O III] $\lambda 4363$ was measured for eight of the $25 \mathrm{H}$ II regions. The nebular

\footnotetext{
2 IRAF is distributed by the National Optical Astronomy Observatories, which are operated by the Association of Universities for Research in Astronomy, Inc., under cooperative agreement with the National Science Foundation.
}

Table 1

Observed $\mathrm{H}$ II region sample

\begin{tabular}{|c|c|c|c|c|}
\hline (1) & $\begin{array}{c}\text { R.A. } \\
(\mathrm{J} 2000.0) \\
(2)\end{array}$ & $\begin{array}{c}\text { Decl. } \\
(\mathrm{J} 2000.0) \\
(3)\end{array}$ & $\begin{array}{c}\mathrm{R} \\
(\mathrm{kpc}) \\
(4)\end{array}$ & other ID \\
\hline 1 . & 13333.53 & $\begin{array}{lll}30 & 41 & 29.8\end{array}$ & 1.83 & NGC 595 \\
\hline$\ldots$ & $\begin{array}{lll}1 & 33 & 39.94\end{array}$ & $\begin{array}{lll}30 & 41 & 07.4\end{array}$ & 1.21 & B 50 \\
\hline $3 \ldots \ldots$ & 13343.69 & $\begin{array}{llll}30 & 40 & 58.1\end{array}$ & 0.87 & B 52 \\
\hline $4 \ldots$ & 13349.65 & $30 \quad 3955.6$ & 0.17 & H 670 \\
\hline $5 \ldots \ldots$ & 13348.39 & $\begin{array}{lll}30 & 39 & 35.7\end{array}$ & 0.22 & B 43b* \\
\hline $6 \ldots \ldots$ & $\begin{array}{lll}133 & 48.17\end{array}$ & $\begin{array}{lll}30 & 39 & 16.4\end{array}$ & 0.22 & B 37 \\
\hline $7 \ldots \ldots$ & 13336.31 & $\begin{array}{lll}30 & 38 & 48.4\end{array}$ & 1.22 & B 1008a \\
\hline $8 \ldots \ldots$ & 13347.78 & $\begin{array}{lll}30 & 38 & 37.6\end{array}$ & 0.30 & В $29^{*}$ \\
\hline$\ldots$ & 13349.42 & $\begin{array}{lll}30 & 38 & 00.2\end{array}$ & 0.42 & B 20a \\
\hline $10 \ldots \ldots$ & 13350.07 & $\begin{array}{lll}30 & 37 & 30.2\end{array}$ & 0.57 & B $16^{*}$ \\
\hline $11 \ldots \ldots$ & 13334.86 & $\begin{array}{lll}30 & 37 & 05.2\end{array}$ & 1.31 & B $33 b^{*}$ \\
\hline $12 \ldots \ldots$ & 13345.48 & $\begin{array}{lll}30 & 36 & 48.5\end{array}$ & 0.74 & B 27a* \\
\hline $13 \ldots \ldots$ & $\begin{array}{lll}134 & 34.78\end{array}$ & $\begin{array}{lll}30 & 35 & 49.7\end{array}$ & 1.71 & B $1^{*}$ \\
\hline $14 \ldots \ldots$ & 13359.32 & $\begin{array}{lll}30 & 35 & 46.5\end{array}$ & 1.55 & B $4 a$ \\
\hline $15 \ldots \ldots$ & 13400.32 & $\begin{array}{lll}30 & 34 & 17.1\end{array}$ & 1.99 & $\mathrm{C} 1 \mathrm{Ab}^{*}$ \\
\hline $16 \ldots \ldots$ & 13400.03 & $\begin{array}{lll}30 & 33 & 56.5\end{array}$ & 2.07 & B 8 Ac \\
\hline $17 \ldots \ldots$ & 13350.23 & $\begin{array}{lll}30 & 33 & 47.5\end{array}$ & 1.62 & B $15 a$ \\
\hline $18 \ldots \ldots$ & 13347.66 & $\begin{array}{lll}30 & 33 & 37.6\end{array}$ & 1.60 & B $18^{*}$ \\
\hline $19 \ldots \ldots$ & $\begin{array}{lll}1 & 33 & 42.91\end{array}$ & $\begin{array}{lll}30 & 33 & 30.1\end{array}$ & 1.58 & B $23^{*}$ \\
\hline $20 \ldots \ldots$ & 13354.13 & $\begin{array}{lll}30 & 33 & 09.6\end{array}$ & 1.96 & B $13 c^{*}$ \\
\hline $21 \ldots \ldots$ & $\begin{array}{lll}1 & 33 & 47.87\end{array}$ & $\begin{array}{lll}30 & 33 & 05.0\end{array}$ & 1.75 & B $17 d$ \\
\hline $22 \ldots \ldots$ & 13359.94 & $\begin{array}{lll}30 & 32 & 45.1\end{array}$ & 2.38 & В 703 \\
\hline $23 \ldots \ldots$ & 13357.43 & $\begin{array}{llll}30 & 32 & 42.3\end{array}$ & 2.25 & M em 43 \\
\hline $24 \ldots \ldots$ & 13346.92 & $\begin{array}{lll}30 & 32 & 35.1\end{array}$ & 1.87 & near C 53 \\
\hline $25 \ldots \ldots$ & 13344.52 & $\begin{array}{lll}30 & 32 & 01.1\end{array}$ & 1.98 & B $1502 a$ \\
\hline
\end{tabular}

Note. - Units of right ascension are hours, minutes and seconds, and units of declination are degrees, arcminutes and arcseconds. Col. (1): H II region identification. Col. (2): Right ascension. Col. (3): Declination. Col. (4): Deprojected galactocentric distance in kpc. Col. (5): Identification from Boulesteix et al. (1974. = B), Hodge et al. [1999. = H), Courtes et al. $(1987=\mathrm{C}$ ) and Magrini et al. $[2000=\mathrm{M})$. Objects with a * symbol in column (5) are in common with Rosolowsky \& Simon 2008.

package in IRAF was used to derive $T_{e}$ for the $\mathrm{O}^{++}$-emitting region, adopting an electron density of $10^{2} \mathrm{~cm}^{-3}$ and the same atomic parameters used by Bresolin et al. (2009). The temperature in the $\mathrm{O}^{+}$-emitting region was estimated from the relation $\mathrm{T}[\mathrm{O} \mathrm{II}]=0.7 \mathrm{~T}[\mathrm{O} \mathrm{III}]+3000 \mathrm{~K}$ (Garnett 1992). The $\mathrm{O}^{++}$ temperatures and the computed $\mathrm{O} / \mathrm{H}$ abundance ratios are presented in columns (10) and (11) of Table 2, respectively.

Ten objects in Table 1 are in common with the work by RS08 (these are identified by the $*$ symbol in column 5). Moreover, NGC 595 (object 1 in Table 1) has also been recently observed with high resolution spectroscopy by Esteban et al. (2009). A comparison with the line fluxes contained in these two publications is presented in Fig. 2. It can be seen that the agreement is generally acceptable, except for target $15(\mathrm{C} 1 \mathrm{Ab})$, for which RS08 measured a much stronger [O III] $\lambda 5007$ flux than the value reported here. It appears likely that the slits used by RS08 and in this work covered different portions of this high-excitation nebula. Fig. 22 indicates that $R_{23}$, which can be used as a metallicity indicator $^{3}$, is generally consistent between the different works. The [O III] $\lambda 4363$-derived oxygen abundances in Fig. 2 $\mathrm{d}$ can differ between this work and RS08 by significant amounts, up to 0.4 dex. The logarithmic extinction $\mathrm{c}(\mathrm{H} \beta)$ was found to be systematically higher by an average of 0.19 dex in comparison with the RS08 values, for objects in common. The comparison with the fluxes and the oxygen abundance published for NGC 595 by Esteban et al. (2009, full symbols in Fig. 2) is excellent. The $\mathrm{c}(\mathrm{H} \beta)$ value determined for this target is also

\footnotetext{
${ }^{3} \mathrm{R}_{23}=([\mathrm{O}$ II $] \lambda 3727+[\mathrm{O}$ III $] \lambda \lambda 4959,5007) / \mathrm{H} \beta$ (Pagel et al. 1979).
} 


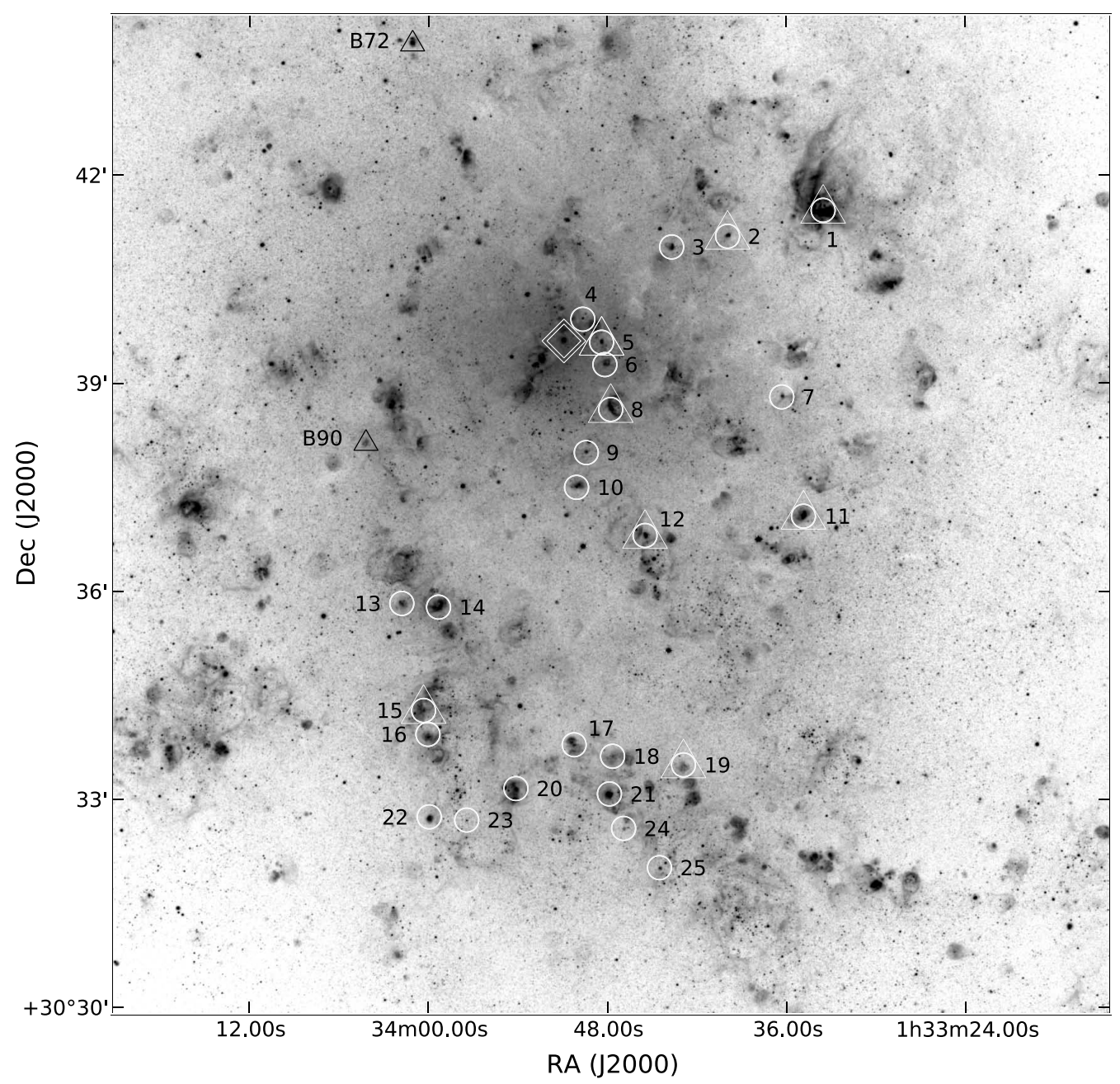

Figure 1. Location of the H II regions studied in this work on a narrow-band $\mathrm{H} \alpha$ image of M33 (taken from the Local Group Galaxies Survey, Massey et al. 2006). Targets where the [O III] $\lambda 4363$ line was detected are marked with triangles. The galaxy center is shown by the diamond symbol. The objects B 72 and B 90 were observed with the Subaru telescope by Bresolin et al. [2010).

in good agreement with their work (0.46 vs. 0.39$)$.

\section{THE RADIAL ABUNDANCE GRADIENT AND ITS SCATTER}

The radial abundance gradient in M33 has been investigated from H II region spectroscopy by several authors in the past few years (see RS08, Magrini et al. 2010, Bresolin et al. 2010 and references therein). The large number of available $\mathrm{H}$ II regions with published line flux data is utilized here to infer the scatter in abundance at a given galactocentric distance. The new Gemini sample was designed to increase the number of observations near the galactic center, where the detection of the auroral lines is most critical for the radial abundance gradient, although problematic even with $8 \mathrm{~m}$-class telescopes.

Line fluxes for $83 \mathrm{H}$ II regions with [O III] $\lambda 4363$ detections (in addition to [O II] $\lambda 3727$ and [O III] $\lambda 5007$ ) have been collected from the following papers: Kwitter \& Aller (1981), Vílchez et al. (1988), Crockett et al. (2006), RS08, Magrini et al. (2010) and Bresolin et al. (2010, Subaru observations). The oxygen abundances were recomputed from the published reddening-corrected emission line fluxes with the same procedure and atomic data used for the Gemini H II region sample. The resulting radial abundance gradient is shown in Fig. 3 where different symbols are used for different data sources. The large scatter of the data points in the diagram, already noticed by RS08 for their sample, is evident: the rms scatter around the least square fit (dashed line) for the full sample is 0.17 dex, apparently larger than what can be accounted for by the measuring uncertainties. However, looking at the distribution of the data points in the central $2.2 \mathrm{kpc}$ of the galaxy, the new Gemini data presented here, together with the Subaru data from Bresolin et al. (2010), display a much lower scatter (0.06 dex, 11 objects), compared to the RS08 data (0.21 dex, 19 objects) pertaining to the same inner portion of the disk. A Kolgomorov-Smirnov test indicates that the probability of obtaining the low scatter measured for the Gemini+Subaru sample from the distribution of the RS08 data points is $<3 \%$. This suggests that the dispersion in Fig. 3 might be related to the quality of the spectroscopic data drawn from the literature, for example to the signal-to-noise $(\mathrm{S} / \mathrm{N})$ ratio of the [O III] $\lambda 4363$ line detections, rather than intrinsic azimuthal variations on scales of the order of a few hundred pc. It can also be seen that the RS08 abundances for the two innermost $\mathrm{H}$ II regions (B 43b and B 29$)$ are significantly lower $(\sim 0.3$ dex) than those measured from the Gemini sample.

To test the effect of the reported quality of the $\lambda 4363$ line detection on the abundance scatter, H II regions for which this line was observed with a $\mathrm{S} / \mathrm{N}<6$ were removed, together with 11 RS08 objects in common with the Gemini and Sub- 
Table 2

Reddening-corrected line fluxes and oxygen abundances

\begin{tabular}{|c|c|c|c|c|c|c|c|c|c|c|}
\hline (1) & $\begin{array}{c}{[\mathrm{O} \text { II] }} \\
3727 \\
(2)\end{array}$ & $\begin{array}{c}{[\mathrm{Ne} \text { III }]} \\
3868 \\
(3)\end{array}$ & $\begin{array}{c}\text { [O III] } \\
4363 \\
(4)\end{array}$ & $\begin{array}{c}\text { H I } \\
4101 \\
(5)\end{array}$ & $\begin{array}{c}\text { [O III] } \\
4959 \\
(6)\end{array}$ & $\begin{array}{c}\text { [O III] } \\
5007 \\
(7)\end{array}$ & $\begin{array}{c}\mathrm{F}(\mathrm{H} \beta) \\
\left(\mathrm{erg} \mathrm{s}^{-1} \mathrm{~cm}^{-2}\right) \\
(8)\end{array}$ & $\begin{array}{c}c(\mathrm{H} \beta) \\
(\mathrm{mag}) \\
(9)\end{array}$ & $\begin{array}{c}T_{e} \\
(\mathrm{~K}) \\
(10)\end{array}$ & $12+\log (\mathrm{O} / \mathrm{H})$ \\
\hline 1. & $169 \pm 17$ & $4.7 \pm 0.5$ & $0.42 \pm 0.04$ & $23 \pm 2$ & $50 \pm 3$ & $150 \pm 9$ & $1.7 \times 10^{-14}$ & 0.46 & $7960 \pm 300$ & $8.42 \pm 0.09$ \\
\hline $2 \ldots$ & $213 \pm 20$ & $7.2 \pm 0.7$ & $0.42 \pm 0.07$ & $25 \pm 2$ & $56 \pm 3$ & $171 \pm 10$ & $2.1 \times 10^{-14}$ & 0.43 & $7710 \pm 410$ & $8.55 \pm 0.12$ \\
\hline $3 \ldots \ldots \ldots$ & $189 \pm 18$ & $4.2 \pm 0.7$ & $\ldots$ & $25 \pm 2$ & $34 \pm 2$ & $99 \pm 6$ & $1.1 \times 10^{-14}$ & 0.51 & $\ldots$ & $\ldots$ \\
\hline $4 \ldots \ldots \ldots$ & $245 \pm 25$ & $4.9 \pm 1.1$ & $\ldots$ & $27 \pm 2$ & $40 \pm 2$ & $118 \pm 7$ & $3.8 \times 10^{-15}$ & 0.72 & $\ldots$ & $\ldots$ \\
\hline$\ldots$ & $169 \pm 16$ & $8.3 \pm 0.8$ & $0.59 \pm 0.08$ & $28 \pm 2$ & $64 \pm 4$ & $194 \pm 11$ & $1.9 \times 10^{-14}$ & 0.67 & $8100 \pm 390$ & $8.45 \pm 0.11$ \\
\hline$\ldots \ldots$ & $195 \pm 20$ & $4.6 \pm 0.8$ & $\ldots$ & $29 \pm 2$ & $36 \pm 2$ & $109 \pm 6$ & $5.4 \times 10^{-15}$ & 0.37 & $\ldots$ & $\ldots$ \\
\hline $7 \ldots \ldots \ldots$ & $275 \pm 26$ & $2.9 \pm 0.7$ & $\ldots$ & $26 \pm 2$ & $27 \pm 2$ & $81 \pm 5$ & $5.7 \times 10^{-15}$ & 0.38 & $\ldots$ & $\ldots$ \\
\hline$\ldots \ldots$ & $181 \pm 18$ & $3.4 \pm 0.4$ & $0.18 \pm 0.04$ & $26 \pm 2$ & $37 \pm 2$ & $112 \pm 7$ & $2.0 \times 10^{-14}$ & 0.29 & $6980 \pm 400$ & $8.59 \pm 0.13$ \\
\hline $9 \ldots$ & $194 \pm 20$ & $\ldots$ & $\ldots$ & $26 \pm 2$ & $17 \pm 1$ & $51 \pm 3$ & $5.3 \times 10^{-15}$ & 0.00 & $\ldots$ & $\ldots$ \\
\hline $10 \ldots$ & $192 \pm 22$ & $\ldots$ & $\ldots$ & $26 \pm 2$ & $22 \pm 1$ & $66 \pm 4$ & $9.1 \times 10^{-15}$ & 0.24 & $\ldots$ & $\ldots$ \\
\hline $11 \ldots \ldots \ldots$ & $206 \pm 20$ & $8.9 \pm 0.9$ & $0.48 \pm 0.12$ & $25 \pm 2$ & $59 \pm 3$ & $177 \pm 10$ & $3.8 \times 10^{-14}$ & 0.77 & $7860 \pm 570$ & $8.52 \pm 0.15$ \\
\hline $12 \ldots$ & $239 \pm 23$ & $2.5 \pm 0.3$ & $0.30 \pm 0.08$ & $26 \pm 2$ & $30 \pm 2$ & $90 \pm 5$ & $2.8 \times 10^{-14}$ & 0.44 & $8300 \pm 660$ & $8.36 \pm 0.16$ \\
\hline $13 \ldots$ & $266 \pm 26$ & $3.9 \pm 0.7$ & $\ldots$ & $26 \pm 2$ & $27 \pm 2$ & $81 \pm 5$ & $9.7 \times 10^{-15}$ & 0.22 & $\ldots$ & $\ldots$ \\
\hline $14 \ldots$ & $197 \pm 19$ & $3.7 \pm 0.6$ & $\ldots$ & $25 \pm 2$ & $46 \pm 3$ & $138 \pm 8$ & $2.0 \times 10^{-14}$ & 0.40 & $\ldots$ & $\ldots$ \\
\hline $15 \ldots$ & $221 \pm 21$ & $46.1 \pm 4.2$ & $2.78 \pm 0.32$ & $25 \pm 2$ & $145 \pm 9$ & $444 \pm 26$ & $6.6 \times 10^{-15}$ & 0.19 & $9880 \pm 520$ & $8.41 \pm 0.11$ \\
\hline $16 \ldots$ & $178 \pm 17$ & $\ldots$ & $\ldots$ & $25 \pm 2$ & $17 \pm 1$ & $52 \pm 3$ & $9.3 \times 10^{-15}$ & 0.00 & $\ldots$ & $\ldots$ \\
\hline $17 \ldots$ & $240 \pm 23$ & $6.4 \pm 0.8$ & $\ldots$ & $26 \pm 2$ & $25 \pm 1$ & $73 \pm 4$ & $1.0 \times 10^{-14}$ & 0.22 & $\ldots$ & $\ldots$ \\
\hline $18 \ldots$ & $238 \pm 23$ & $27.7 \pm 3.2$ & $\ldots$ & $26 \pm 2$ & $95 \pm 6$ & $284 \pm 17$ & $4.0 \times 10^{-15}$ & 0.16 & $\ldots$ & $\ldots$ \\
\hline $19 \ldots$ & $262 \pm 25$ & $49.4 \pm 4.6$ & $3.31 \pm 0.41$ & $27 \pm 2$ & $183 \pm 11$ & $539 \pm 32$ & $5.0 \times 10^{-15}$ & 0.08 & $9810 \pm 530$ & $8.51 \pm 0.11$ \\
\hline $20 \ldots$ & $242 \pm 23$ & $8.1 \pm 0.8$ & $\ldots$ & $26 \pm 2$ & $45 \pm 3$ & $134 \pm 8$ & $2.1 \times 10^{-14}$ & 0.41 & $\ldots$ & $\ldots$ \\
\hline $21 \ldots$ & $243 \pm 23$ & $3.2 \pm 0.4$ & $\ldots$ & $25 \pm 2$ & $20 \pm 1$ & $62 \pm 4$ & $3.3 \times 10^{-14}$ & 0.18 & $\cdots$ & $\cdots$ \\
\hline $22 \ldots$ & $219 \pm 21$ & $7.5 \pm 1.0$ & $\ldots$ & $24 \pm 2$ & $61 \pm 4$ & $180 \pm 11$ & $1.5 \times 10^{-14}$ & 0.55 & $\ldots$ & $\ldots$ \\
\hline $23 \ldots$ & $248 \pm 24$ & $\ldots$ & $\ldots$ & $26 \pm 2$ & $1 \pm 0$ & $4 \pm 0$ & $1.5 \times 10^{-15}$ & 0.16 & $\ldots$ & $\ldots$ \\
\hline $24 \ldots$ & $224 \pm 23$ & $\ldots$ & $\ldots$ & $25 \pm 4$ & $28 \pm 2$ & $85 \pm 5$ & $8.7 \times 10^{-16}$ & 1.00 & $\ldots$ & $\ldots$ \\
\hline $25 \ldots \ldots \ldots$ & $253 \pm 24$ & $\cdots$ & $\cdots$ & $25 \pm 2$ & $12 \pm 1$ & $34 \pm 2$ & $3.1 \times 10^{-15}$ & 0.15 & $\cdots$ & $\cdots$ \\
\hline
\end{tabular}

Note. - The line fluxes are in units of $\mathrm{H} \beta=100 . \mathrm{F}(\mathrm{H} \beta)$ in column (8) is the measured $\mathrm{H} \beta$ flux, uncorrected for extinction.

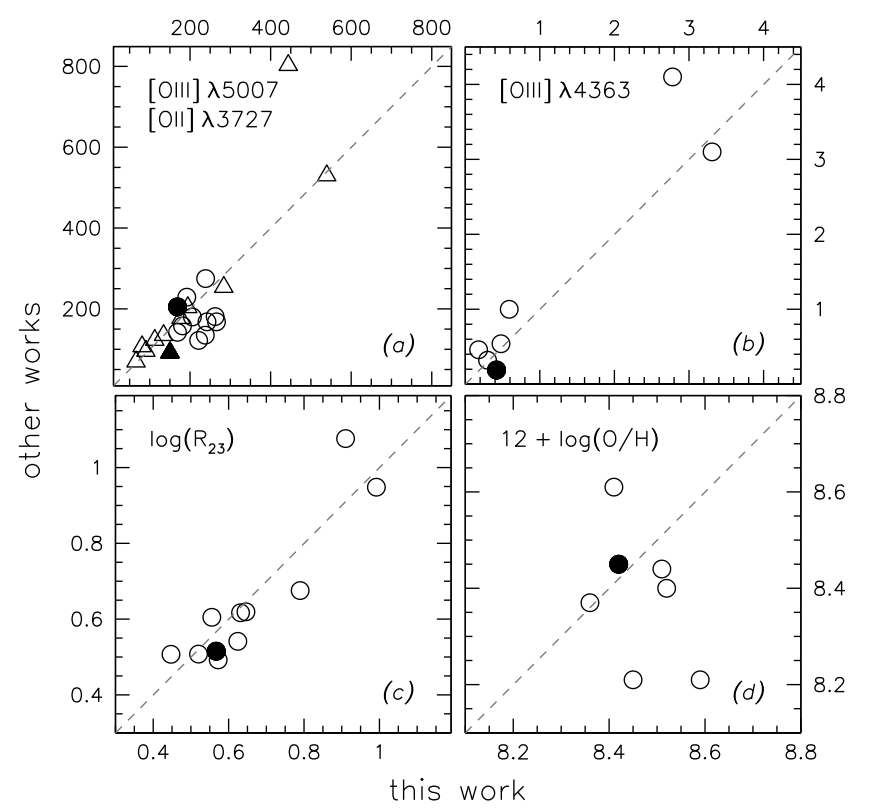

Figure 2. Comparison between the data obtained for this work and results from the literature for: (a) the [O II] $\lambda 3727$ (circles) and [O III] $\lambda 5007$ (triangles) reddening-corrected fluxes (in units of $\mathrm{H} \beta=100$ ); $(b)$ the [O III $] \lambda 4363$ fluxes; (c) $\log \left(\mathrm{R}_{23}\right)$; (d) $12+\log (\mathrm{O} / \mathrm{H})$. The dashed line represents the one-toone correlation line. The open and full symbols refer to the comparison with RS08 and Esteban et al. (2009. NGC 595 only), respectively.

aru observations done by the author. The result is shown in Fig. 4. The overall scatter is only slightly reduced to 0.13 , still a factor of two larger than what is measured in the inner two kpc from the author's data alone. However, the ap- parent abundance peak shown by the RS08 data at $1.5 \mathrm{kpc}$ from the galaxy center, as well as the lack of high $\mathrm{O} / \mathrm{H}$ values in the central kpc present in the RS08 sample, are now gone. The radial oxygen abundance gradient measured from this subsample (short-dashed line in Fig. 4) is:

$$
12+\log (\mathrm{O} / \mathrm{H})=8.48( \pm 0.04)-0.042( \pm 0.010) \mathrm{R}_{\mathrm{kpc}}
$$

where $\mathbf{R}_{\mathrm{kpc}}$ is the galactocentric distance in kpc. A $2 \sigma$ iterative clipping was also applied to the data, providing a comparable result, but with smaller errors (long-dashed line):

$$
12+\log (\mathrm{O} / \mathrm{H})=8.50( \pm 0.02)-0.045( \pm 0.006) \mathrm{R}_{\mathrm{kpc}} \text {. }
$$

Because of the removal of several low-metallicity $\mathrm{H}$ II regions in the central two kpc belonging to the RS08 sample the slope of the gradient given above is larger than the one determined by $\operatorname{RS} 08\left(-0.027 \pm 0.012 \mathrm{dex} \mathrm{kpc}{ }^{-1}\right)$. The intercept value is also larger, 8.50 vs. 8.36. The measurement of the new Gemini $T_{e}$-based abundances in the inner two kpc of M33 thus shows that $(i)$ it is possible that the real abundance scatter in this galaxy is not as large as claimed by RS08; (ii) the radial abundance gradient in M33 behaves 'normally', as seen in other galaxies, with the metallicity increasing smoothly towards the center, without indications of a central dip, which has been ascribed by Magrini et al. (2010) to a selection bias. The interpretation given here is that it might have to do instead with errors in the measurements. Magrini et al. (2010) have also proposed that using only giant H II regions to determine the abundance gradient yields a steeper gradient than using the full sample, regardless of luminosity. This is not 
Table 3

Abundances for additional $\mathrm{H}$ II regions

\begin{tabular}{ccc}
\hline \hline ID & $\begin{array}{c}\mathrm{R} \\
(\mathrm{kpc})\end{array}$ & $12+\log (\mathrm{O} / \mathrm{H})$ \\
$(1)$ & $(2)$ & $(3)$ \\
\hline B 72 & 1.18 & $8.46 \pm 0.09$ \\
B 90 & 1.40 & $8.47 \pm 0.08$ \\
B 302 & 2.07 & $8.44 \pm 0.08$ \\
\hline \multicolumn{3}{l}{ Note. - Data from the Subaru observations of } \\
Bresolin et al.|2010.
\end{tabular}

confirmed by the abundance analysis carried out in the current paper. From the same nine giant H II regions they used, the abundance gradient is:

$$
12+\log (\mathrm{O} / \mathrm{H})=8.50( \pm 0.10)-0.040( \pm 0.024) \mathrm{R}_{\mathrm{kpc}},
$$

which is essentially the same as found above.

The latter result, based on only nine regions, is useful to offer an explanation as to how various authors have come up with different values for the slope of the gradient in M33. The $1 \sigma$ range for the slope in Eq. 3 virtually encompasses all of the $\mathrm{HII}$ region-based values published in the past twenty years, from $-0.012 \pm 0.011 \mathrm{dex} \mathrm{kpc}^{-1}$ (Crockett et al. 2006) to $-0.05 \pm 0.01 \mathrm{dex} \mathrm{kpc}^{-1}$ (Vílchez et al. 1988, after scaling to the M33 distance adopted here and removing the central H II regions), several of which have been determined from a similarly small number of $\sim 10 \mathrm{H}$ II regions. (The central abundances from Vílchez et al.1988 are model-based, and lead to a steep gradient in the inner portion of the disk; however, the direct measurements based on the new auroral line detections do not support such high abundance values). A significantly larger number of data points, well distributed in radius, is required to reduce the uncertainty in the slope (Dutil \& Roy 2001, Bresolin et al. 2009).

As a final remark, the spatial distribution of the $\mathrm{H}$ II regions in the central parts of M33 is very similar between the Gemini and the RS08 samples, with the majority of the objects located along the spiral arms. Although it is implicit in the distribution of the Gemini and Subaru data points in Fig. 4 it is worth emphasizing that pairs of $\mathrm{H}$ II regions with nearly coincident galactocentric distances, but situated almost diametrically opposite each other relative to the galaxy center have identical oxygen abundances, within the errors. This is the case for B 90 vs. B 50, B 72 vs. B $33 \mathrm{~b}$ and B 302 vs. C 1 Ab (the abundances for the three H II regions studied with Subaru by Bresolin et al. 2010 are summarized in Table 3 for convenience). This implies that the ISM of M33 is well mixed azimuthally over scales of at least $4 \mathrm{kpc}$.

\subsection{The abundance scatter determined from $R_{23}$}

Despite the fact that the subsample of $\mathrm{H}$ II regions selected from the literature with a reported detection of [O III] $\lambda 4363$ better than $6 \sigma$ displays only a slight improvement in the rms scatter around the exponential gradient compared to the full sample, the much smaller dispersion from the Gemini+Subaru observations by the author suggests that the real abundance fluctuations in M33 are actually smaller and comparable to what is observed in other well-studied spiral galaxies. To test this assertion, and to show that it not simply a consequence of the relatively small number of objects considered (11) in

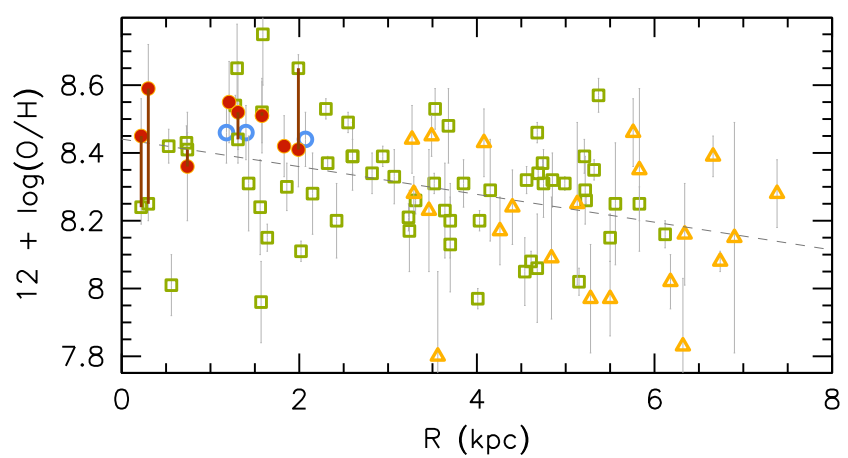

Figure 3. Radial oxygen abundance gradient in M33, using [O III] $\lambda 4363$ based abundances from this paper (full circles), Bresolin et al. [2010, open circles), Rosolowsky \& Simon (2008 squares) and other sources in the literature (triangles). The least square fit to the full dataset is shown by the dashed line. Vertical segments connect objects in common between the Gemini and RS08 samples.

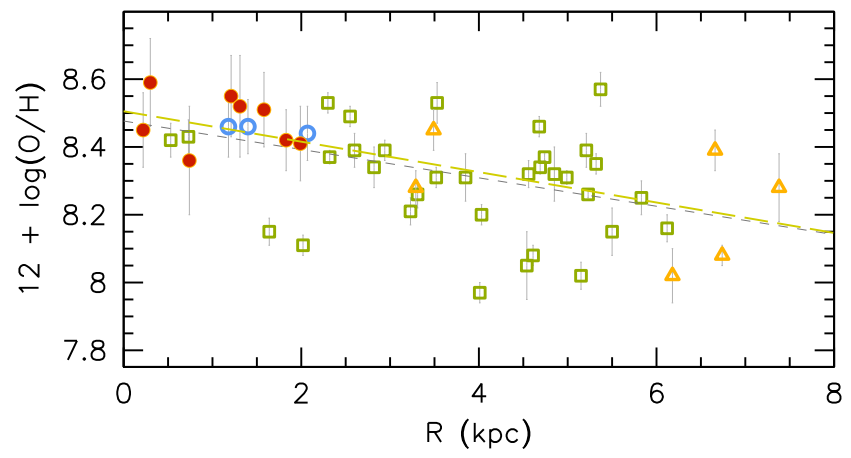

Figure 4. Same as Fig. 3 with sample reduced to the highest signal-to-noise [O III] $\lambda 4363$ detections, as explained in the text. The lines represent fits to all the data points shown (short dashes) and to a smaller sample obtained by means of a $2 \sigma$ iterative clipping procedure (long dashes).

the inner disk alone, the oxygen abundances for the $\mathrm{H}$ II region sample shown in Fig. 3 were computed with the $\mathrm{R}_{23}$ abundance indicator, which uses only strong emission lines, so that the resulting abundances are largely independent of the random errors affecting the much weaker [O III] $\lambda 4363$ auroral lines, and which propagate through the chemical abundance measurement. Despite the large systematic uncertainties affecting $\mathrm{R}_{23}$ and other strong-line methods, as shown by the comparison of $T_{e}$-based abundances with different calibrations of strong-line indicators (e.g. Bresolin et al. 2009), the abundance scatter should be comparable to what high-quality auroral line detections indicate (somewhat larger dispersions can be expected from the dependence of $\mathrm{R}_{23}$ on the ionization conditions of the different H II regions, see Kennicutt \& Garnett 1996). In fact, as mentioned earlier, Bresolin et al. (2009) measured the abundance gradient of NGC 300 from [O III] $\lambda 4363$ line detections in $28 \mathrm{H} \mathrm{II} \mathrm{regions,} \mathrm{finding} \mathrm{an} \mathrm{rms}$ scatter of 0.05 dex. Comparable values, between 0.06 and $0.08 \mathrm{dex}$, are measured from the same data set when the abundances are obtained from a variety of strong-line abundance indicators.

The $\mathrm{R}_{23}$ calibration of McGaugh (1991), as given in Kuzio de Naray et al. (2004), was adopted, since it has the advantage over other available calibrations of accounting for the nebular gas excitation, as given by the $y=\log (\mathrm{O} 3 / \mathrm{O} 2)$ parameter, with $\mathrm{O} 3=[\mathrm{O}$ III $] \lambda \lambda 4959,5007 / \mathrm{H} \beta$ and $\mathrm{O} 2=[\mathrm{O}$ II $] \lambda 3727 / \mathrm{H} \beta$. The upper branch calibration was used, considering the published 
$[\mathrm{N}$ II $] / \mathrm{H} \alpha$ values, and the position of the targets in a $\mathrm{R}_{23}$ vs $\mathrm{O} / \mathrm{H}$ plot (presented in Sec. 47).

The $\mathrm{O} / \mathrm{H}$ abundance ratio thus calculated is shown as a function of radial distance in Fig. 5 for the same H II regions presented in Fig. 3 A subsample, shown with full symbols, was created by selecting objects with (a) S/N ([O III] $\lambda 4363)>5 ; \quad$ (b) $[\mathrm{Ne} \mathrm{III]} \lambda 3868 / \mathrm{H} \beta<0.45 ; \quad$ (c) $[\mathrm{O}$ III $] \lambda 5007 / \mathrm{H} \beta<5$; (d) $\log \mathrm{R}_{23}<0.90$. These criteria will be justified and discussed at length in Sec. 4, and serve to isolate $\mathrm{H}$ II regions that are not affected by extremely hard ionization fields, or objects for which the $\mathrm{R}_{23}$-based abundances could be very uncertain. H II regions that do not satisfy these conditions are shown either with open symbols (low $\mathrm{S} / \mathrm{N}$ in the [O III] $\lambda 4363$ line), double open symbols (strong [Ne III] and [O III] lines) and crossed symbols (large $\mathrm{R}_{23}$ ).

The dashed line in Fig. 5 represents a least square fit to the data belonging to the subsample thus obtained, limiting the fit to galactocentric distances smaller than $5 \mathrm{kpc}$ (to avoid possible biases when reaching down to metallicities near the turnover region of $\mathrm{R}_{23}$, as explained in Sec. 47. The result is

$$
12+\log (\mathrm{O} / \mathrm{H})=8.82( \pm 0.02)-0.033( \pm 0.005) \mathrm{R}_{\mathrm{kpc}} \text {. }
$$

The slope is slightly shallower than the value obtained from the auroral lines, although the two are still consistent within the $1 \sigma$ errors. It is worth recalling here the dependence of the slope of galactic radial abundance gradients on the method chosen to measure the oxygen abundances for their H II regions (e.g. Bresolin et al. 2009). As an example, applying the widely-used $\mathrm{R}_{23}$ calibrations by Tremonti et al. (2004) and Zaritsky et al. (1994) yields slopes of $-0.044 \pm 0.006$ dex kpc ${ }^{-1}$ and $-0.051 \pm 0.007 \mathrm{dex} \mathrm{kpc}{ }^{-1}$, respectively. Moreover, as mentioned earlier, there are important systematic discrepancies in the metallicities derived from strong-line methods calibrated from theoretical model grids (as in the case of the $\mathrm{R}_{23}$ calibration by McGaugh 1991 adopted here) and the auroral-line method. This explains the large offset between the intercepts of the regression lines shown in Fig. 3 and Fig. 5. However, this has virtually no effect on the derivation of the abundance scatter.

The most important result to note is that the rms scatter of the data shown with full symbols in Fig. 5 is only 0.05 dex, which is virtually the same value found from the [O III $\lambda$ 4363-based abundances for the innermost $\mathrm{H}$ II regions observed with Gemini and Subaru. Changing the $\mathrm{R}_{23}$ calibration has virtually no effect, as the rms scatter remains between 0.05 and 0.07 dex throughout the disk of the galaxy. Following RS08, the least-square method by Akritas \& Bershady (1996), which computes the intrinsic scatter of the data, was applied to the $\mathrm{R}_{23}$-based abundances, confirming that the observed scatter is due only to measurement errors. The data therefore indicate the absence of real azimuthal variations in the oxygen abundances.

It should be pointed out that the RS08 H II regions located in the central two kpc, which showed a very large scatter in the $T_{e}$-based abundance plot of Fig. 3, are now in excellent agreement with the new Gemini data, and have a comparably small abundance scatter. It should also be added that the selection based on the $\mathrm{S} / \mathrm{N}$ of the [O III] $\lambda 4363$ line has little effect on the measured rms scatter, but it helps to retain the targets with the best determined values of the $[\mathrm{O}$ II $] \lambda 3727$ and [O III] $\lambda \lambda 4959,5007$ lines, used to define the $\mathrm{R}_{23}$ indicator.

The main conclusion that can be drawn from the analysis

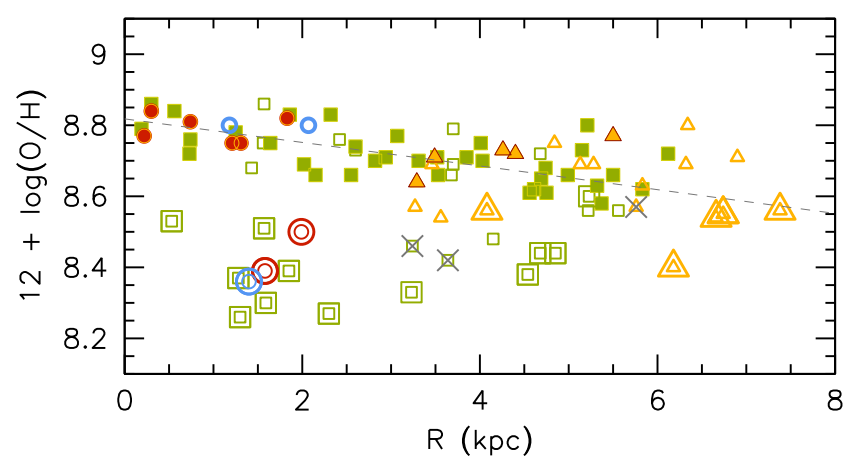

Figure 5. Radial abundance gradient in $\mathrm{M} 33$ measured from the $\mathrm{R}_{23}$ indicator. $\mathrm{H}$ II regions from the new Gemini observations (red circles), the Subaru data of Bresolin et al. (2010 bue circles), the sample of RS08 (squares) and other data from the literature (triangles) are included. The dashed line represents the linear fit to the subsample defined in the text and shown by full symbols. Double-lined symbols are used for H II regions with very high [Ne III] and [O III] fluxes. Crossed symbols are used for additional objects with $\log \left(\mathrm{R}_{23}\right)>0.90$. The remaining open symbols represent H II regions with low $\mathrm{S} / \mathrm{N}$ in the $[\mathrm{O}$ III] $\lambda 4363$ line.

of the $\mathrm{R}_{23}$-based abundances, supported by the discussion of the $T_{e}$-based results in the inner two kpc, is that the abundance scatter in M33 is small (0.05-0.06 dex), comparable to the measuring errors, and similar in magnitude to what is observed in other well-studied spiral galaxies, such as NGC 300. We can therefore speculate that the significant intrinsic scatter seen in the $T_{e}$-based abundances of M33 from the RS08 sample is due to measurement errors in the weak [O III] $\lambda 4363$ auroral line. From Fig. 4 and 5 it is also possible to claim that the oxygen abundance reaches its peak at the center of the galaxy, as is typically the case for spiral galaxies, and therefore that there is no evidence for the off-centered abundance distribution suggested by Magrini et al. (2010).

\section{SYSTEMATIC EFFECTS OF A HARD IONIZING RADIATION ON THE $R_{23}$ ABUNDANCES}

In Fig. 5 a number of targets were found to lie systematically below the sequence of $\mathrm{H}$ II regions defining the $\mathrm{R}_{23}$ based abundance gradient in M33. In particular, in the inner part of the disk the oxygen abundances of several $\mathrm{H}$ II regions are $\sim 0.3$ to 0.5 dex below the regression representing the abundance gradient, and appear to belong to a secondary sequence of data points that merges into the main one at a galactocentric distance of about $7 \mathrm{kpc}$.

In order to interpret the origin for this discrepancy, and justify the exclusion of these targets from the $R_{23}$-based abundance gradient analysis, the $T_{e}$-based oxygen abundances of the same H II regions included in Fig. 5 are shown as a function of $\mathrm{R}_{23}$ in Fig. 6 . In this diagram, a number of extragalactic $\mathrm{H}$ II regions with high-quality metallicity determinations, based on the [O III] $\lambda 4363$ auroral line, are also included (small dots). They were drawn from the following sources:
NGC 300:
M101:
NGC 2403:
Bresolin et al. (2009)
IC 1613:
H II galaxies:

\begin{tabular}{l|}
\hline Kennicutt et al. (2003) \\
\hline Garnett et al. (1997) \\
\hline Esteban et al. $(2009)$ \\
\hline Bresolin et al. \\
\hline Izotov et al. (1906) \\
\hline Guseva et al. (2009) \\
\hline Esteban et al. (2009) \\
\hline
\end{tabular}
M31, NGC 2363, NGC 1741 Esteban et aI. (2009) NGC 4395, NGC 4861 


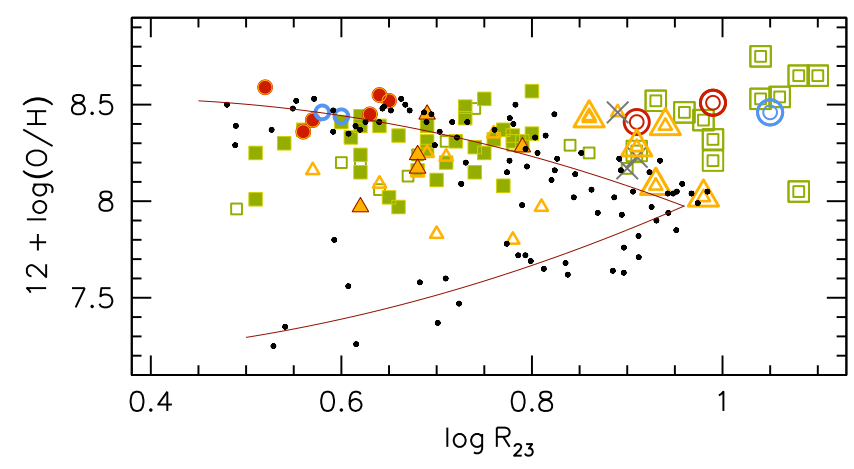

Figure 6. Oxygen abundances derived from the [O III] $\lambda 4363$ auroral line as a function of $\log \left(\mathrm{R}_{23}\right)$. A sample of $\mathrm{H}$ II regions with high-quality measurements in different spiral and irregular galaxies (small dots) have been used to schematically define the upper and lower branches of $\mathrm{R}_{23}$ (shown by the two intersecting curves). H II regions from the new Gemini observations (red circles), the Subaru data of Bresolin et al. (2010 bue circles), the sample of RS08 (squares) and other data from the literature (triangles) are included. The symbols used are the same as in Fig. 5

These data illustrate the well-known bi-valued nature of $\mathrm{R}_{23}$, consisting of two $\mathrm{H}$ II region sequences, intersecting around $12+\log (\mathrm{O} / \mathrm{H})=8.0$. The curves in Fig. 6 are polynomial fits shown to guide the eye in recognizing the two branches. The $\mathrm{H}$ II region data for M33 are plotted with the same symbols used in Fig. 5. It can be immediately seen that the majority of the most discrepant points in Fig. 5, identified by the large doubled-lined symbols, lie at large $\mathrm{R}_{23}$ values and above the intersection between the upper and lower branches in Fig. 6. Very large $R_{23}$ values $\left(\log R_{23}>1\right.$ ) are relatively rare among extragalactic $\mathrm{H}$ II regions, and are encountered in highexcitation nebulae with large ionization parameters. A fraction of such objects show spectral features associated with the presence of massive stars emitting particularly hard ionizing radiation, such as early WN (WNE) stars (Crowther 2007). It is thus not surprising that the H II regions where RS08 have identified nebular He II $\lambda 4686$ line emission (I.P. $=54.4 \mathrm{eV}$ ) are located, together with additional discrepant points, in the upper right corner of the $\mathrm{R}_{23} \mathrm{vs}$. $\mathrm{O} / \mathrm{H}$ plot. Invariably in this zone of the diagram, even when the He II emission remains undetected or the ionizing sources cannot be unequivocally identified, the high-excitation lines [Ne III] $\lambda 3868$ (I.P. $=41$ $\mathrm{eV})$ and $[\mathrm{O} \mathrm{III}] \lambda 5007(\mathrm{I} . \mathrm{P} .=35 \mathrm{eV})$ are particularly strong.

\subsection{H II regions with large [Ne III] and [O III] line fluxes}

The large double-lined symbols in Fig. 6 were used to identify $\mathrm{H}$ II regions where $\mathrm{Ne}_{3868}=[\mathrm{Ne}$ III $] \lambda 3868 / \mathrm{H} \beta>0.45$ or $\mathrm{O}_{5007}=\left[\mathrm{O}_{\mathrm{III}}\right] \lambda 5007 / \mathrm{H} \beta>5$. The use of both line ratios is somewhat redundant, since the two are strongly correlated (the relative values of the criteria adopted for $\mathrm{Ne}_{3868}$ and $\mathrm{O}_{5007}$ are consistent with the trend found between the two line ratios from the enlarged $\mathrm{H}$ II region sample analyzed here). The choice of the adopted selection criteria is purely empirical and somewhat arbitrary, and was guided by the knowledge of the line fluxes observed in nearby nebulae ionized by WNE stars. For instance, in RCW 5 and RCW 48 (Milky Way), and N57 C and N138 D, B (LMC), Kennicutt et al. (2000) measured $\mathrm{Ne}_{3868}>0.56$ and $\mathrm{O}_{5007}>5.8$, while, for example, for the giant $\mathrm{H}$ II region 30 Dor in the LMC, ionized by a cluster of $\mathrm{O}$ stars, $\mathrm{Ne}_{3868}=0.40$ and $\mathrm{O}_{5007}=4.3$.

Typical upper branch $\mathrm{H}$ II regions in other galaxies are observed with $\mathrm{Ne}_{3868}<0.40$ and $\mathrm{O}_{5007}<4.0$, and highly excited $\mathrm{H}$ II regions, such as \#15 (C 1Ab) and \#19 (B 23) in Table 1 easily stand out among objects with much lower $\mathrm{Ne}_{3868}$ and
$\mathrm{O}_{5007}$ values. However, it should be recalled that high $\mathrm{Ne}_{3868}$ and $\mathrm{O}_{5007}$ values, also exceeding the limits adopted above, can be encountered in lower branch $\mathrm{H}$ II regions (low metallicities), or near the turnaround between the two $R_{23}$ branches. This explains why a few $\mathrm{HII}$ regions at galactocentric distances $>6 \mathrm{kpc}$ were rejected in Fig. 5 by the criteria adopted above, even though their abundances can agree with the abundance gradient defined by the remaining regions. In fact, the $\mathrm{H}$ II regions at large radial distances are near or at the turnaround, as Fig. 6 shows. For this reason the linear fit to the $\mathrm{R}_{23}$-based abundances has been limited to galactocentric distances smaller than $5 \mathrm{kpc}$.

It should be noted that not all of the discrepant objects in M33 contain known Wolf-Rayet stars, and also that the presence of WNE stars in an extragalactic H II region does not necessarily imply peculiarly large $\mathrm{Ne}_{3868}$ and $\mathrm{O}_{5007}$ values. This occurs only if the nebular ionization is dominated by the radiation emitted by the WN star(s), and the dilution resulting from the presence of $\mathrm{O}$ stars is small. This agrees with the observation that the total $\mathrm{H}$ ionizing flux output of the discrepant M33 H II regions analyzed here, estimated from the $\mathrm{H} \alpha$ luminosity, is consistent with the ionization being provided by only 1-2 WNE stars. What could be a little surprising is finding such a large fraction $(\sim 20 \%$ of the nebulae with [O III] $\lambda 4363$ detections) of highly excited $\mathrm{H}$ II regions in M33. To the author's knowledge, for no other galaxy has an $\mathrm{H}$ II region population been insofar found with such a large percentage of nebulae having $\log \left(\mathrm{R}_{23}\right)>1$. On the other hand, the typical luminosity of $\mathrm{H}$ II regions selected in extragalactic abundance studies is generally significantly higher than that of the majority of those observed in M33. Consequently, their integrated spectra would be less affected by the presence of a few sources of hard radiation amid a large number of ionizing $\mathrm{O}$ stars. In fact, none of the M33 H II regions with a total luminosity $\mathrm{L}(\mathrm{H} \alpha)>10^{38} \mathrm{erg} \mathrm{s}^{-1}$ (adopting fluxes published by Hodge et al. 2002 and Relaño \& Kennicutt 2009) for which a spectrum is available displays notable levels of excitation. The only (marginal) exception in the upper branch regime is IC $131\left(\mathrm{Ne}_{3868}=0.47, \mathrm{O}_{5007}=3.9\right.$, Vílchez et al. 1988). The reason in this case is possibly connected to the fact that, besides containing three WN stars, this giant $\mathrm{H}$ II region has also a peculiarly hard and extended X-ray emission, whose source is at present unexplained (Tüllmann et al. 2009).

\subsection{Excitation diagnostic diagrams}

To clarify the effect of the presence of WNE stars or other sources of hard ionizing radiation on the emission line spectra of H II regions, Fig. 7 shows a [N II] $\lambda 6583 / \mathrm{H} \alpha$ vs. $[\mathrm{O} \mathrm{III}] \lambda 5007 / \mathrm{H} \beta$ diagnostic diagram (Baldwin et al. 1981), in which M33 H II region data from the literature (including the recent work on He II-emitting $\mathrm{H}$ II regions by Kehrig et al. 2010) are shown together with a small number of Galactic and extragalactic H II regions (Kennicutt et al. 2000; Bresolin et al. 2009) containing identified WNE stars (star symbols). The WR ring nebulae G2.4 + 1.4 (WO1), NGC 2359 (WN4), NGC 3199 and S308 (WN5) from Esteban et al. (1993) are also shown (crosses). Additional H II region data for spiral and irregular galaxies from the literature (Bresolin et al. 1999, 2005, and those shown as small dots in Fig. 6) are included for comparison. Nebulae lying above the curve (taken from Kauffmann et al. 2003) are considered to be ionized by a harder ionizing field than provided by $\mathrm{O}$ stars. In this plot the selection of objects with $\mathrm{O}_{5007}>5$ for the discrepant points in Fig. 6 corresponds to the shaded area, assuming that 


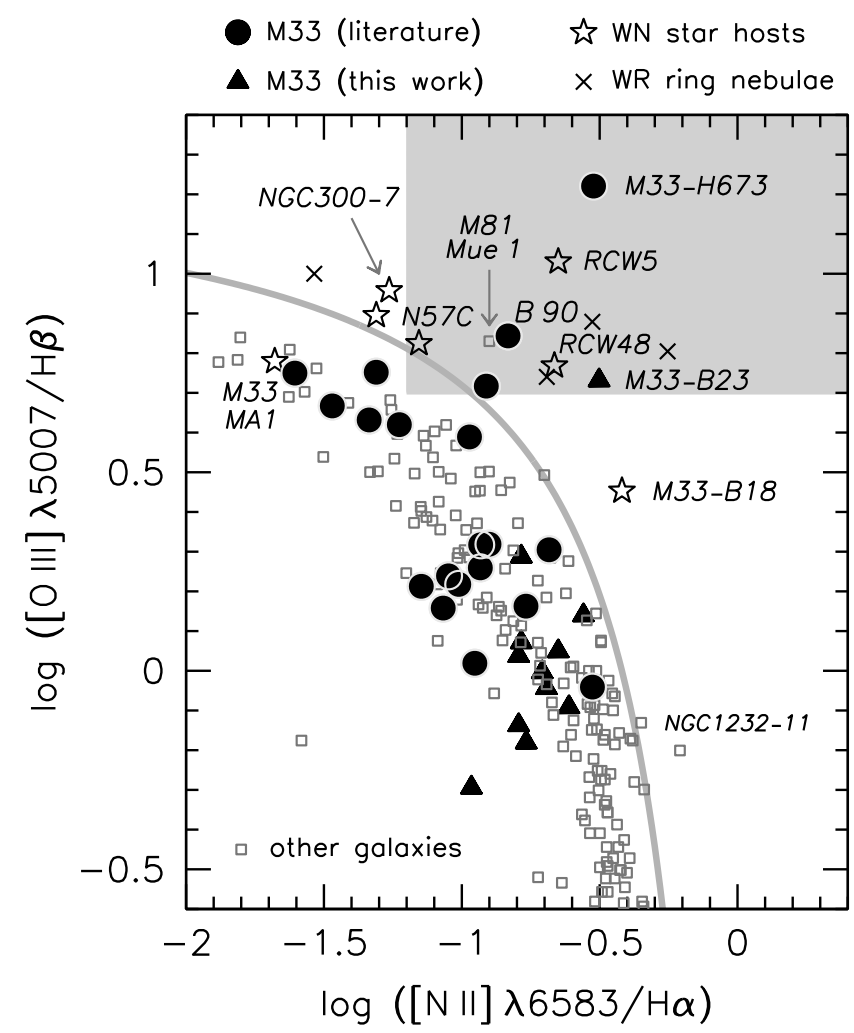

Figure 7. The $\left[\mathrm{O}_{\mathrm{III}}\right] / \mathrm{H} \beta$ vs. $[\mathrm{N}$ II $] / \mathrm{H} \alpha$ diagnostic diagram for $\mathrm{H}$ II regions in M33 from this work (triangles) and from the literature (full circles). H II regions in the Milky Way and the Large Magellanic Cloud (Kennicutt et al. 2000), NGC 300 (Bresolin et al. 2009) and M33 (this work and Kehrig et al. 2010) hosting WN stars are included (star symbols; most are identified). WR ring nebulae from Esteban et al. (1993) are shown with crosses. Additional nebulae contained in spiral and irregular galaxies are taken from Bresolin et al. (1999. 2005) and other sources (open squares) to help discern the H II region excitation sequence. The curve is the boundary between star-forming and AGN-dominated galaxies defined by Kauffmann et al. (2003). The shadowed area includes high-excitation $\mathrm{H}$ II regions in the upper $\mathrm{R}_{23}$ branch having $[\mathrm{O} \mathrm{III}] / \mathrm{H} \beta>5$.

$\log \left(\left[\mathrm{N}_{\mathrm{II}}\right] \lambda 6583 / \mathrm{H} \alpha\right)>-1.2$ on the upper branch (Kewley \& Ellison 2008).

It can be seen that the presence of WNE stars can shift H II regions into the grey area and above the boundary line. Still, there can be low-metallicity (small $[\mathrm{N} \mathrm{II}] / \mathrm{H} \alpha$ ratio) nebulae containing WNE stars (e.g. MA 1 in M33) whose position in the diagram agrees with the excitation sequence defined by the control sample. Moreover, some of the high-excitation $\mathrm{H}$ II regions have no clear detection of associated hot stars (e.g. H673 in M33, Kehrig et al. 2010). For 13 objects in the Gemini sample the spectral coverage extended to sufficiently long wavelengths that the $[\mathrm{N}$ II] $\lambda 5755$ could be measured. From the knowledge of the electron temperature or assuming $T_{e}=10^{4} \mathrm{~K}$ the strength of the [N II] $\lambda 6583$ was estimated using the temden program in IRAF. These objects were then added to Fig. 7 as triangles. One of these, region B 18, contains a WN star (Massey \& Johnson 1998) clearly detected in the Gemini spectrum from its broad He II $\lambda 4686$ emission line. This $\mathrm{H}$ II region lies to the right of the boundary drawn in the diagram. For another, region B 23, situated into the shaded area, no possible match in the Wolf-Rayet catalog by Massey \& Johnson (1998) could be found (the same is true for B 90, observed with the Subaru telescope), although its excitation, as measured from the $[\mathrm{O} \mathrm{III}] / \mathrm{H} \beta$ line ratio, is higher than for B 18 .
The position of some of the deviating H II regions can be linked to the presence of shocks, which can enhance the strength of the [N II] emission line. This is almost certainly the case for region 11 in NGC 1232 (Bresolin et al. 2005 identified near the bottom of Fig. 7), whose spectrum shows strong [O I] $\lambda 6300$ and [S II] $\lambda \lambda 6717,6731$ emission (thus being a supernova remnant candidate). For other H II regions the situation is less clear. For example, H673 in M33 has moderately strong [N II] and [S II] fluxes, but no [O I] emission has been reported by Kehrig et al. (2010). Nitrogen selfenrichment is also an option to explain the large [N II] flux, since these authors report $\log (\mathrm{N} / \mathrm{O})=-0.74 \pm 0.28$, versus a typical $\log (\mathrm{N} / \mathrm{O})=-1.2$ for $\mathrm{H}$ II regions in M33 (Bresolin et al. 2010).

Fig. 7 shows that also Münch 1, an outlying object in M81, studied in some detail by Garnett \& Shields (1987), is among the restricted number of high-excitation extragalactic $\mathrm{H}$ II regions contained within the shaded area $\left(\mathrm{Ne}_{3868}=0.46\right.$, $\mathrm{O}_{5007}=6.9$ ). Given its location in the BPT diagram, it is possible that future high-sensitivity spectra of this region will reveal nebular He II and/or WN-related stellar emission features.

\subsection{H II regions with large $R_{23}$ values}

In Fig. 5 it was decided to remove from the final sample also the HII regions for which $\log \left(\mathrm{R}_{23}\right)>0.9$. The crossed symbols in the plot represent the three H II regions that were removed based only on this condition (rather than poor $\mathrm{S} / \mathrm{N}$, or large [Ne III] and [O III] line fluxes). The condition on $\mathrm{R}_{23}$ is added because the turnover in the oxygen abundance vs. $R_{23}$ relation occurs around $\log \left(R_{23}\right) \simeq 1$. It is well known that here the $\mathrm{R}_{23}$ indicator is a poor abundance diagnostic, because a wide range of abundances corresponds to a narrow range of indicator values (McGaugh 1991). Studies of radial abundance gradients in spiral galaxies should therefore exclude $\mathrm{H}$ II regions in the turnover region in order to avoid abundance biases or large measuring uncertainties. It should also be noted that the selection based on $\mathrm{R}_{23}$ effectively removes virtually all of the targets with high [Ne III] and [O III] fluxes. The previous discussion, however, is helpful to understand what kind of objects systematically deviate from the $\mathrm{R}_{23}$ vs. $\mathrm{O} / \mathrm{H}$ trend of Fig. 6 .

In conclusion, $\mathrm{H}$ II regions ionized by uncommonly hard ionizing radiation (such as that generated by the presence of early WN stars, but other poorly constrained processes are also at work) are shifted to higher $\mathrm{R}_{23}$ values at constant $\mathrm{O} / \mathrm{H}$ abundance ratios compared to 'normal' H II regions in a diagram like the one shown in Fig. 6. As a consequence, $\mathrm{R}_{23}$ would systematically underestimate the abundances for these objects, as observed in Fig. 5 because the upper branch bends to smaller $\mathrm{O} / \mathrm{H}$ values with increasing $\mathrm{R}_{23}$. This also explains the secondary sequence seen at the bottom of this figure, progressively diverging from the gradient regression line with decreasing radial distance (equivalent to increasing $R_{23}$ ). The empirical emission-line criteria outlined above allow to discriminate quite effectively against such discrepant $\mathrm{H}$ II regions.

\section{SUMMARY}

New GMOS/Gemini spectra of $25 \mathrm{H}$ II regions located in the central two kpc of M33 have been analyzed. The oxygen abundances have been derived from the [O III] $\lambda 4363$ auroral line for eight of the targets. The scatter in the radial abundance 
gradient has been investigated by combining existing samples of $\mathrm{H}$ II regions in this galaxy with the new data. The main conclusions of this work are summarized as following:

(1) the scatter in the oxygen abundance in the central two kpc, as derived from the auroral line measurements for the new Gemini sample, is approximately $0.06 \mathrm{dex}$, much lower than the value of $\sim 0.21$ dex obtained in the same inner portion of the disk from the H II regions observed by Rosolowsky \& Simon (2008).

(2) the oxygen abundances estimated from the $\mathrm{R}_{23}$ metallicity indicator for a large sample of $\mathrm{H}$ II regions assembled from the literature in combination with the new observations yield a comparably small scatter (0.05-0.07 dex) over the whole optical disk of M33. This dispersion can be explained simply by the measuring uncertainties.

(3) no evidence is found for significant intrinsic azimuthal variations in the $\mathrm{H}$ II region metallicity distribution in M33 on scales from $\sim 100 \mathrm{pc}$ to a few $\mathrm{kpc}$, as well as for a displacement of the abundance peak from the galaxy center. This result rules out large anomalies in the mixing of the gas.

(4) a considerable fraction of the M33 H II regions with auroral line detections show spectral features revealing sources of hard ionizing radiation (He II emission, large [Ne III] and [O III] line fluxes). In some cases these can be identified with early WN stars, but several cases exist where no obvious source has been identified. For these nebulae the oxygen abundances derived from $R_{23}$ are systematically underestimated by large factors. However, it is straightforward to identify these highly excited $\mathrm{H}$ II regions from their large $\mathrm{R}_{23}$ values and their position in the excitation diagnostic diagrams. Thus, abundance gradients derived for other spiral galaxies hosting such objects would still be correct, once these peculiar nebulae are removed from the abundance analysis based on the strong-line metallicity indicator.

FB gratefully acknowledges the support from the National Science Foundation grants AST-0707911 and AST-1008798, and thanks the referee, Cesar Esteban, for comments that helped to improve the manuscript. The Gemini observations used in this paper were obtained under program GN-2009BQ-5.

\section{Facility: Gemini:Gillett (GMOS)}

\section{REFERENCES}

Akritas, M. G., \& Bershady, M. A. 1996, ApJ, 470, 706

Baldwin, J. A., Phillips, M. M., \& Terlevich, R. 1981, PASP, 93, 5

Boissier, S., \& Prantzos, N. 1999, MNRAS, 307, 857

Boulesteix, J., Courtes, G., Laval, A., Monnet, G., \& Petit, H. 1974, A\&A, 37, 33

Bresolin, F. 2008, in The Metal-Rich Universe, ed. G. Israelian \& G. Meynet, 155

Bresolin, F., Gieren, W., Kudritzki, R., Pietrzyński, G., Urbaneja, M. A., \& Carraro, G. 2009, ApJ, 700, 309

Bresolin, F., Kennicutt, Jr., R. C., \& Garnett, D. R. 1999, ApJ, 510, 104

Bresolin, F., Pietrzyński, G., Urbaneja, M. A., Gieren, W., Kudritzki, R.-P., \& Venn, K. A. 2006, ApJ, 648, 1007

Bresolin, F., Schaerer, D., González Delgado, R. M., \& Stasińska, G. 2005, A\&A, 441, 981
Bresolin, F., Stasińska, G., Vílchez, J. M., Simon, J. D., \& Rosolowsky, E. 2010, MNRAS, 404, 1679

Courtes, G., Petit, H., Petit, M., Sivan, J., \& Dodonov, S. 1987, A\&A, 174, 28

Crockett, N. R., Garnett, D. R., Massey, P., \& Jacoby, G. 2006, ApJ, 637, 741

Crowther, P. A. 2007, ARA\&A, 45, 177

de Vaucouleurs, G., de Vaucouleurs, A., Corwin, Jr., H. G., Buta, R. J.,

Paturel, G., \& Fouque, P. 1991, Third reference catalogue of bright galaxies (Springer-Verlag Berlin Heidelberg New York)

Dutil, Y., \& Roy, J.-R. 2001, AJ, 122, 1644

Edmunds, M. 2005, Astronomy and Geophysics, 46, 4.12

Elmegreen, B. G. 1998, in Astronomical Society of the Pacific Conference Series, Vol. 147, Abundance Profiles: Diagnostic Tools for Galaxy History, ed. D. Friedli, M. Edmunds, C. Robert, \& L. Drissen, p. 278

Esteban, C., Bresolin, F., Peimbert, M., García-Rojas, J., Peimbert, A., \& Mesa-Delgado, A. 2009, ApJ, 700, 654

Esteban, C., Smith, L. J., Vilchez, J. M., \& Clegg, R. E. S. 1993, A\&A, 272, 299

Freedman, W. L., et al. 2001, ApJ, 553, 47

Fu, J., Hou, J. L., Yin, J., \& Chang, R. X. 2009, ApJ, 696, 668

Garnett, D. R. 1992, AJ, 103, 1330

Garnett, D. R., \& Shields, G. A. 1987, ApJ, 317, 82

Garnett, D. R., Shields, G. A., Skillman, E. D., Sagan, S. P., \& Dufour, R. J. 1997, ApJ, 489, 63

Gogarten, S. M., et al. 2010, ApJ, 712, 858

Guseva, N. G., Papaderos, P., Meyer, H. T., Izotov, Y. I., \& Fricke, K. J. 2009, A\&A, 505, 63

Hodge, P. W., Balsley, J., Wyder, T. K., \& Skelton, B. P. 1999, PASP, 111, 685

Hodge, P. W., Skelton, B. P., \& J., A. 2002, An Atlas of Local Group Galaxies (Dordrecht: Kluwer)

Hook, I. M., Jørgensen, I., Allington-Smith, J. R., Davies, R. L., Metcalfe, N., Murowinski, R. G., \& Crampton, D. 2004, PASP, 116, 425

Izotov, Y. I., Thuan, T. X., \& Lipovetsky, V. A. 1994, ApJ, 435, 647

Kauffmann, G., et al. 2003, MNRAS, 346, 1055

Kehrig, C., et al. 2010, ArXiv e-prints

Kennicutt, R. C., Bresolin, F., \& Garnett, D. R. 2003, ApJ, 591, 801

Kennicutt, R. C., \& Garnett, D. R. 1996, ApJ, 456, 504

Kennicutt, Jr., R. C., Bresolin, F., French, H., \& Martin, P. 2000, ApJ, 537, 589

Kewley, L. J., \& Ellison, S. L. 2008, ApJ, 681, 1183

Kuzio de Naray, R., McGaugh, S. S., \& de Blok, W. J. G. 2004, MNRAS, 355,887

Kwitter, K. B., \& Aller, L. H. 1981, MNRAS, 195, 939

Magrini, L., Corradi, R. L. M., Mampaso, A., \& Perinotto, M. 2000, A\&A, 355,713

Magrini, L., Stanghellini, L., Corbelli, E., Galli, D., \& Villaver, E. 2010, A\&A, 512, A63

Massey, P., Bianchi, L., Hutchings, J. B., \& Stecher, T. P. 1996, ApJ, 469, 629

Massey, P., \& Johnson, O. 1998, ApJ, 505, 793

Massey, P., Olsen, K. A. G., Hodge, P. W., Strong, S. B., Jacoby, G. H.,

Schlingman, W., \& Smith, R. C. 2006, AJ, 131, 2478

Matteucci, F., \& Francois, P. 1989, MNRAS, 239, 885

McGaugh, S. S. 1991, ApJ, 380, 140

Pagel, B. E. J., Edmunds, M. G., Blackwell, D. E., Chun, M. S., \& Smith, G. 1979, MNRAS, 189, 95

Relaño, M., \& Kennicutt, R. C. 2009, ApJ, 699, 1125

Rosolowsky, E., \& Simon, J. D. 2008, ApJ, 675, 1213

Roy, J.-R., \& Kunth, D. 1995, A\&A, 294, 432

Scalo, J., \& Elmegreen, B. G. 2004, ARA\&A, 42, 275

Seaton, M. J. 1979, MNRAS, 187, 73P

Tremonti, C. A., et al. 2004, ApJ, 613, 898

Tüllmann, R., et al. 2009, ApJ, 707, 1361

Vila-Costas, M. B., \& Edmunds, M. G. 1992, MNRAS, 259, 121

Vílchez, J. M., Pagel, B. E. J., Díaz, A. I., Terlevich, E., \& Edmunds, M. G. 1988, MNRAS, 235, 633

Williams, B. F., Dalcanton, J. J., Dolphin, A. E., Holtzman, J., \& Sarajedini, A. 2009, ApJ, 695, L15

Yin, J., Hou, J. L., Prantzos, N., Boissier, S., Chang, R. X., Shen, S. Y., \& Zhang, B. 2009, A\&A, 505, 497

Zaritsky, D., Elston, R., \& Hill, J. M. 1989, AJ, 97, 97

Zaritsky, D., Kennicutt, Jr., R. C., \& Huchra, J. P. 1994, ApJ, 420, 87 